\title{
Part Per Million
}

National Cancer Institute

\section{Source}

National Cancer Institute. Part Per Million. NCI Thesaurus. Code C48523.

A unit of proportion equal to $10 \mathrm{E}-6$. 